\title{
In vitro biological effects of magnetic nanoparticles
}

\author{
LI Yan, CHEN ZhongWen \& GU Ning* \\ State Key Laboratory of Bioelectronics, Jiangsu Key Laboratory of Biomaterials and Devices, School of Biological Science and Medical \\ Engineering, Southeast University, Nanjing 210096, China
}

Received January 14, 2012; accepted March 12, 2012; published online July 10, 2012

\begin{abstract}
Magnetic nanoparticles (MNPs) have great potential for a wide use in various biomedical applications due to their unusual properties. It is critical for many applications that the biological effects of nanoparticles are studied in depth. To date, many disparate results can be found in the literature regarding nanoparticle-biological factors interactions. This review highlights recent developments in this field with particular focuses on in vitro MNPs-cell interactions. The effect of MNPs properties on cellular uptake and cytotoxicity evaluation of MNPs were discussed. Some employed methods are also included. Moreover, nanoparticle-cell interactions are mediated by the presence of proteins absorbed from biological fluids on the nanoparticle. Many questions remain on the effect of nanoparticle surface (in addition to nanoparticle size) on protein adsorption. We review papers related to this point too.
\end{abstract}

magnetic nanoparticles, biological effects, interactions, cytotoxicity, cellular uptake

Citation: $\quad$ Li Y, Chen Z W, Gu N. In vitro biological effects of magnetic nanoparticles. Chin Sci Bull, 2012, 57: 3972-3978, doi: 10.1007/s11434-012-5295-8

The current development of magnetic nanoparticles (MNPs) requires a better understanding of its biological effects. A great deal of work has been conducted to study MNPsbiological factors interactions. Many researchers bend themselves to in vitro cell-based assessment. In this work, the current knowledge of how the in vitro cultured cells can interact with the exposed colloid MNPs is discussed as well as the effect of nanoparticle size and surface properties on cell responses. The specific cell line selected for in vitro assay is intended to model a response or phenomenon likely observed or sensitized by particles in vivo. Here, the frequent lack of consistency or predictability between in vitro models and in vivo observations, which is because of the different biological conditions particles exposed to and the changed cell phenotype against primary cell types, is out of our consideration. As we have known, cell monocultures as measured by in vitro assays rarely react in such isolated pathways in native tissues comprosed of multiple, dynamically communicative cell types that produce non-linear and correlated response to foreign materials.

*Corresponding author (email: guning@ seu.edu.cn)
Adsorption of proteins onto the nanoparticle surface happens immediately after particles come in contact with a biological fluid, and it is the proteins associate with nanoparticles, leading to a protein "corona" which defines the biological identity of the particle. Here, we also try to review some current work on associated protein "corona" when nanoparticles were incubated in biological medium.

\section{Overview of magnetic nanoparticles}

MNPs have been attracting much attention because of their prospective biomedical applications, either as contrast agents for magnetic resonance imaging (MRI) [1], heating mediators for hyperthermia [2], introcellular cell labeling and tracking [3], or as drug/gene delivery carriers [4]. Several materials including iron oxide $\left(\gamma-\mathrm{Fe}_{2} \mathrm{O}_{3}\right.$ or $\left.\mathrm{Fe}_{3} \mathrm{O}_{4}\right)$, cobalt, nickel and manganese ferrites, metals and metal alloys such as $\mathrm{Fe}$ and $\mathrm{FePt}$, have been developed [5]. In all of them, iron oxide nanopartilces are used more commonly than others because they could be simply synthesized, easily modified and functionalized, and biocompatible. In fact, some 
iron oxide-based MNPs (e.g. Endorem and Feridex) have been approved to use clinically by US FDA. MNPs mentioned in the following text mean iron oxide-based MNPs without special indication.

Iron oxide nanoparticles can be synthesized using a variety of techniques, such as aqueous co-precipitation process, high-temperature decomposition of organometallic precursors, etc. The former technique is the easiest and most common, and the process is initiated by mixing an iron salt with polymer surfactants under alkaline conditions. The precise $\mathrm{pH}$ value in the solution and the used surfactant play the predominant role in determining the nanoparticle properties. The latter has been used to precise control the size and shape of nanoparticles by changing the precursors, surfactants and solvents. It could be capable of producing uniform spherical $\mathrm{Fe}_{3} \mathrm{O}_{4}$ nanoparticles with a size variation of $<2 \mathrm{~nm}$ and diameters ranging from 4 to about $22 \mathrm{~nm}$ [5].

To ensure the colloidal stability under physiological conditions and enhance the functionality, iron oxide nanoparticles synthesized need to be efficiently coated with various types of chemistry modifiers including polymers, dendrimers, organic acids, nature polysaccharides, inorganic matrixes, etc. [6]. This is undertaken either during or after synthesis. As a typical example, nanoparticles encapsulated in a hydrophobic polymer shell could be prepared as a nanodevice for transporting a whole spectrum of molecules, including drugs. The encapsulation ensures that the shell is not washed off in hydrophilic media, what would result in sedimentation and aggregation of the magnetic core particles. At the same time, high magnetite contents and uniform distribution of magnetite in the polymer can be achieved. And the functional groups introduced by the polymer shell also offer the opportunity for further functionalization with modifiers like functional ligands [7], organic dyes [8], permeation enhancers (like cell penetrating peptides, CPPs) [9] or antibodies [10] to imbue them with specific biological functionalities. Embedding iron oxide nanoparticle into the polymer shell can also be synthesized for multifunctional devices serving as contrast agents for either MRI or ultrasound imaging [11]. In addition to polymers, liposomes, polyelectrolyte multilayer microscapsules or micelles are typical examples of organic matrixes that can be used for encapsulation of iron oxide nanoparticles [5]. Inorganic matrixes such as silica [12] or gold [13] could also be available for the synthesis of such core-shell or embedment structure. Figure 1 illustrates MNPs with different surface functionalities.

\section{Protein corona associated with nanoparticles}

The differential proteins bound to the surface of nanoparticles can influence the tissue distribution, cellular uptake, and biological effects of nanoparticles. Moreover, in a biological circumstance, proteins associate with nanoparticles,



Figure 1 Illustration of MNPs with different surface functionalities.

and the amount and presentation of the proteins on the surface rather than the particles themselves that is the cause of numerous biological responses. It is this outer layer of proteins (protein "corona") that is actually seen by the biological cell, organ or barrier, and leads to the in vivo responses [14]. Dissecting the composition of protein corona in a given biological fluid may allow predictions of the particle's fate regarding its interactions with specific cell types and surface receptors as well as predictions of its half-life in the body [15]. Thus, various studies have been conducted to reveal the knowledge of particle-guided protein fingerprints and their dependence on nanomaterial properties.

Currently, several techniques are available for isolating nanoparticle-protein complexes, including equilibrium dialysis, size-exclusion chromatography, and microfiltration as being referred by Tenzer et al. [16]. More recently, separation by centrifugation has been selected as common approach as it can easily be used routinely, be reproducible, and requires relatively little material $[16,17]$. For the analysis of the composition of protein corona, SDS-polyacrylamide gel electrophoresis (SDS-PAGE) followed by liquid chromatography mass spectrometry (LC-MS) of digested peptides are used, combining with in silico analysis [16-19]. Also, several spectroscopic techniques (dynamic light scattering, UV-visible, plasmon resonance light scattering) are employed to inspect the dynamic proteins-nanoparticle interactions [20].

Types of nanoparticles were chosen as targeted objects to try to understand the influence of particle size [16], surface roughness [21], surface charge [22], surface chemistries [23] on the composition of the corresponding protein corona formed in a given biological fluid. The referred properties have been suggested to play a significant role in determining the detailed coronas. However, the observations are not always consistent. Studies addressing the influence of different physico-chemical parameters reported various outcomes. And no distinct mechanisms explaining proteinspecific binding have emerged from these studies. For example, some investigators found that particle size predomi- 
nantly affects the protein corona quantitatively rather than qualitatively [16]. In contrast, other studies using similar polymer, gold, or other metal nanoparticles reported not only significant quantitative but also qualitative sizedependent changes in the obtained protein fingerprints [23]. Although no mechanisms have been shown so far explaining why size alone should either allow or completely abolish binding of certain proteins.

Besides size, the influence of other particle characteristics on the composition of associated protein corona also addressed various outcomes. For surface charge, modulating the surface charge of polystyrene nanoparticles significantly influenced the composition of the corona [23]. Also, negatively charged silica nanoparticles were reported to preferentially adsorb proteins with $\mathrm{pI}<7$, displaying a negative charge at $\mathrm{pH} 7.3$, i.e. the $\mathrm{pH}$ present in plasma preparations [16]. In contrast, other investigations found out an increase in plasma protein absorption with surface charge density without affecting the overall protein profile [24]. In our study, MNPs functionalized with silanes bearing various functional groups including amino group, carboxylic group, and short-chain poly(ethylene glycol) (PEG) were incubated in cell culture medium (PRMI 1640 plus 10\% fetal calf serum). Silanes-MNPs with various functional groups had different adsorption capacity to proteins in the cell culture medium, as inferred by UV-visible spectroscopy using Coomassie blue fast staining method, about $4.8 \%$ of proteins in $10 \mathrm{~mL}$ cell culture medium could be adsorbed onto

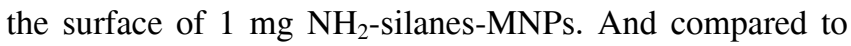
$\mathrm{NH}_{2^{-}}$and $\mathrm{COOH}$-silanes-MNPs, the amount of protein adsorbed by PEG-silanes-MNPs was negligible, indicating protein repellent property of PEG despite its low molecular weight and short chain used in this study [25].

Knowledge of rates, affinities, and stoichiometries of protein association with, and disassociation from, nanoparticles is also studied using simple model systems, plasma and in vitro cell culture medium to understand the nature of the particle surface seen by the functional machinery of cells $[20,26]$. Besides the human plasma or simple protein model systems, differential cytosolic fluids are also used as incubation liquids [17].

The controversial outcomes might be because of different experiment conditions and techniques which were used for protein isolation, separation and identification. As we aware, some proteins may be below the detection limit of the employed methods. With the remarkable development of detection techniques, the underlying mechanisms for such some how unexpected observations, which certainly include hydrophobic/hydrophilic as well as electrostatic interactions, would be resolved gradually. And these finds are helpful for us to scientifically understand, characterize and possibly some additionally emphasize on standards for the surfaces of nanoparticles including magnetic nanoparticles for the future need.

\section{Cellular uptake and processing}

Studies of cellular uptake have attracted much attention as either a safe and efficient localization of MNPs into the cytosol may be critical in some applications such as phototherapy, intracellular imaging, and gene or intracellular drug delivery or it should be avoided as in the extracellular implications. The internalization through endocytosis (the process of uptake of macromolecules into cells by enclosing them in membrane vesicles) upon contact with the cell membrane can occur through a variety of mechanisms which has been reviewed by Verma et al. [15]: (1) phagocytosis, (2) pinocytosis, (3) macropinocytosis, (4) clathrin-mediated endocytosis, and (5) caveolae-mediated endocytosis.

The size of MNPs has been found to greatly influence their cellular uptake. For the uptake routes, phagocytosis is the uptake of particles larger than $500 \mathrm{~nm}$, often triggered by particle opsonization and subsequent receptor-mediated activation of F-actin-driven pseudopods that engulf the particle in a cytoplasmic phagosome, while pinocytosis usually occurs at the sites of clathrin-coated pits and involves the passage of particles smaller than $200 \mathrm{~nm}$ [27]. Cellular uptake amount of nanoparticles is also influenced by the nanoparticle size. In a study by Huang et al. [28], the uptake of 30-120 nm polyvinylpyrrolidone (PVP) coated iron oxide nanoparticles was investigated in RAW264.7 mouse macrophage cells. It was found that the cellular uptake was heavily dependent upon size, with the uptake of particles of $37>65>8>23 \mathrm{~nm}$, which indicated that $37 \mathrm{~nm}$ is the optimal size for cell uptake. A very important factor that should be considered is that even though nanoparticles display a certain size after synthesis, during the in vitro studies they might aggregate into vastly different shapes and sizes that may dictate the outcome and interpretation of results.

Surface charge plays a significant role in the internalization of nanoparticles. It has been well-known that positively charged MNPs were adsorbed more efficiently on the negatively charged cell surface and consequently showed greater internalization than that of neutral and negatively charged MNPs. We have investigated the interactions of three MNPs: dimercaptosuccinic acid (DMSA) coated- (DMSA@MNPs, negative charge), chitosan coated- (CS@MNPs, positive charge) and the agglomerates formed by aggregation between DMSA@MNPs and CS@MNPs (CS-DMSA@MNPs, positive charge) with KB cells (oral squamous carcinoma cell) [29]. The results showed that the cellular uptake of the two positively charged MNPs was higher than that of negatively charged MNPs, and the higher cellular uptake of CS-DMSA@MNPs compared with CS@MNPs, which may result from the high surface charge and large agglomerate size, was detected too. Also in this study, the cellular uptake of MNPs was dependent on the incubation time and nanoparticles concentration. Due to the high affinity of positive CS@MNPs to cells, which leads to efficient cell labeling, a 
novel CS@MNPs covalently attached fluorescent dye for high-efficient cellular imaging serving both as magnetic resonance contrast agents for MRI and optical probes for intravital fluorescence microscopy was further developed by our group [30].

Meanwhile, there has been evidence of uptake of negatively charged particles despite the unfavorable interaction between the particles and the negatively charged cell membrane. As an example, carboxyl-functionalized poly(amidoamine) (PAMAM) dendrimer-stabilized iron oxide nanoparticles have been shown to be taken up into human epithelial carcinoma cells presumably either through pinocytosis or via direct diffusion through the cell membrane [31]. The cellular uptake of DMSA@MNPs in different cell lines was found in many studies including our study in which smooth muscle cells were employed [32]. The internalization of negatively charged nanoparticles may occur through nonspecific binding and clustering of the particles on the scarce cationic sites on the plasma membrane and their subsequent endocytosis.

Compared to charged MNPs, particles coated with a neutral ligand such as poly(ethylene glycol) PEG produced negligible aggregation in cell-culture conditions and reduced nonspecific uptake by macrophage cells as well as low uptake by the liver and spleen. According to this, we have developed dense PEG coated MNPs, which exhibit great application potential in tumor imaging as MRI contrast agents targeting [33].

Besides charge, surface properties such as hydrophilic/ hydrophobic, chemical group or composition may have great influence on MNPs cellular uptake. These properties are commonly given by the chemistries or biomolecules which were used to modify or functionalize nanoparticles. The utilization of cell-penetrating or cell-fusogenic biological ligands conjugated to nanoparticles can help in the fusion of NPs with cell membranes and their uptake into the cytoplasm. These cell-membrane-penetration motifs can chaperon the cargo inside the cell into the cytosol or nucleus. Take the HIV-1 derived tat peptide as an example, following the initial work by Weissleder's group [34] that successfully delivering $41 \mathrm{~nm}$ dextran-coated SPIOs conjugated to tat, resulting in over a hundredfold increase in lymphocyte internalization, other original studies with subsequent in vivo work focused on using tat peptide sequences as tools to enhance cell uptake $[35,36]$. In addition to tat, different modifs including Arg-Gly-Asp (RGD), nuclear-localization signal (NLS) peptides, allatostatin 1, PLL, arginine-rich peptides, and virus proteins have been used for effective cellular delivery of nanoparticles as reviewed by Saha et al. [37]. Many such biological motifs seem to have positively charged residues (assisted by hydrophobic residues) for efficient cellular internalization that correlates very well with the behavior of positively charged organically functionalized nanoparticles [38]. However, it did not affect in the similar manner all the time. Some of these mo- tifs also have an amphipathic surface structure or some may negatively charged. Oligonucleotides, despite of their negative surface charge, can be used as coating modifs which help nanopartilces readily taken up by mouse endothelial cells, and the uptake was dependent on the density of oligonucleotide loading on the nanoparticles surface, with higher densities providing greater uptake [39]. This may due to the interface between adsorbed serum proteins on the particles surface through electrostatic and hydrophobc complementarity with the cell membrane, which revealed the importance of protein adsorption on nanoparticles described in last section.

To better understand the mechanisms of nanoparticle-cell membrane interactions, several in silico studies have been performed by our group. The coarse-grained molecular dynamics simulations aimed at nanoparticle's size effect on its translocation across a dipalmitoylphosphatidylcholine (DPPC) bilayer showed that the interaction of nanoparticles induces the structural variations of membranes, the larger the nanoparticles are, the more space they need to cross the bilayer, and the more significant changes the bilayer shows. The calculation base on the free energy in this study showed that the size of nanoparticles affects the translocation time differently, suggesting that the size has significant impacts on its translocation across the lipid bilayer [40]. Results of coarse-grained molecular dynamics simulations indicated that a hydrophobic nanoparticle can result in the inclusion into the DPPC bilayer, whereas a semihydrophilic nanoparticle is only found to adsorb into the membrane because of the potential substantial energy barrier of particle wrapping, implicating that the endocytosis-like mechanism is an energy-mediated process [41]. Thermodynamics of charged nanoparticle adsorption on charge-neutral membranes was also simulated [42]. The electrostatic attraction improves the adhesion of a charged nanoparticle to the membrane. With the increase of electrostatic energy, a charged nanoparticle can be almost fully wrapped by the membrane. And the nonspecific adsorption of charged nanoparticles has significant effects on the physical characteristic of the membrane. The adsorption of cationic nanoparticles induces the local disordered transition in the adhering region of the membrane, whereas nanoparticles of negative charge induce the formation of the high ordered region in fluid bilayer. The thermodynamic quantities obtained may explain this process based on the Helfrich theory, indicating that the driving force of the wrap originates from the gain in electrostatic energy at the cost of the elastic energy of biomembranes.

Although the mechanisms of endocytosis and the effect of many nanoparticles properties on cellular uptake have been studied extensively, there have still remained a lot of unknowns. For example, the cell-specific uptake of MNPs and the cellular uptake kinetics in different cell lines should be studied systematically. 


\section{Cytotoxicity evaluation}

Recently, it has been shown that despite the initial belief in the non-cytotoxic properties of MNPs, the physicochemical properties of nanoparticles [15] and the high intracellular concentrations of MNPs were required for some implications such as efficient MRI and cell label poses serious threats on cell homeostasis. Thus, the possible toxicity of MNPs with cells and the understanding of the mechanism is becoming an essential focus to ensure the safe use of MNPs in biomedical implications. More recently, much effort has been devoted to investigating the cytotoxicity of MNPs or just confirming the biocompatibility of synthesized MNPs with different surface coatings. Different surface-modified MNPs, including those coated by dextran [43-45], heparin [46], DMSA [29,32], APTS [32], GLU [32], protamine [47], lipid [44,45], PEG and their derivatives [33], silica [12], as well as bare MNPs [48], were evaluated. A series of targeting cell lines were used in vitro for testing including phagocytic [49], neural [48], hepatic [47], epithelial [49], stem and progenitor cells [50], immune and blood cells [49,51] and various cancer cell lines [48].

Several main assay categories, including cellular uptake, cell viability, reactive oxygen species (ROS) production, cell morphology phenotyping, and cell stress are central themes in the testing of influence of nanopartilces on cells, which was reviewed previously [27]. Among them, cellular uptake may play a predominant role in the other cell responses. So we discussed it separately in last section. In this section, we discuss the other cell responses.

Assays of cell viability (live versus dead cells)/proliferation (cell-cycle progression) in a sample provide gross estimates of the cell response to a treatment and are frequently used as an overall biocompatibility assessment criterion. A number of routine, dye-based cell viability/proliferation assays including neutral red, trypan blue, and EDU, formazan-based assays (MTT, MTS, WST) are available for this assessment.

Various reports revealed close links between cell viability/proliferation and nanoparticle size, concentration, surface properties and surface functionalization, which may result from the close links between cellular uptake and these parameters. Although the internalization nanoparticles are not the only factor which can influence cell viability/proliferation, and nanoparticles adsorbed on the cell surface or just the exit of nanoparticles in the cell culture medium may have effects on the cell viability or their proliferation, it has been shown by several groups that high intracellular concentrations of MNPs diminished the viability/proliferation of cultured cells. It was reported that positive superparamagnetic iron oxide nanoparticles (SPIONs- $\mathrm{NH}_{2}$ ) display lower cell viabilities in comparison to negative particles (SPIONs-COOH), and the toxicity amounts of bare SPIONs are higher than those of other coated SPIONs, which was investigated by MTT, in all the three employed human cell
lines-HCM (heart), BE-2-C (brain), and 293T (kidney) [52]. And the reason for the lower cell viability of SPIONs- $\mathrm{NH}_{2}$ might relate to their significant and deep cellular uptake amounts. This effect was also confirmed by our group that the enhancement intracellular uptake of protamine modified MNPs led to the lower particles concentration level at which a significant impairment of treated SMMC-7721 (human hepatoma cell line) cells viability did appear [47]. In another study, four types of iron oxide particles: Resovist (coated with carboxydextran), Endorem (coated with dextran), very small organic particles (coated with citrate), and magnetoliposomes (MLs, coated with lipids) were incubated with murine $\mathrm{C} 17.2$ neural progenitor cells and human blood outgrowth epithelial cells. Cell-cycle progression assessment showed a clear reduction in both cell lines proliferation, the cell doubling time increased compared with untreated control cells. This study also revealed the nanoparticle concentration-denpendent effects on cell proliferation for Resovist and Endorem as the cells appear to recover when the particles have been diluted [45].

Besides evaluating with only one or few kinds of cell in most studies, reliable comprehensive studies to systematically evaluate the cytotoxicity of MNPs on multiple cell lines from different tissues and species were also performed $[43,44,49,53]$. Some of these studies demonstrated that the cytotoxicity of various MNPs is cell-specific. For example, three kinds of cells, i.e. asrocyte, renal cells NRK and rat liver cells BRL 3A, would not obviously be affected by the dextran-stabilized MNPs up to the concentration at 128 $\mu \mathrm{g} / \mathrm{mL}$, while apoptosis was significantly observed for periphery blood mononuclear wells (PBMC) with high concentration particles (64 and $128 \mu \mathrm{g} / \mathrm{mL}$ ) [43]. The conclusion ascribed by these studies could well explain the contradictory results on the toxicity of particles when the exact same nanoparticles were interacted with various cells reported by plenty of literature.

In addition to the direct outcome showed by cell viability/proliferation, assays of ROS (effectors), was also used to deeply understand the cytotoxicity mechanisms of nanopartilces. ROS are products of normal metabolism, at physiological low levels, ROS function as "redox messengers" in intracellular signaling and regulation, whereas excess ROS represents a oxidative stress condition, in which, ROS production overcomes the antioxidant protective ability of the cell, thus generates unfavorable biological effects (inducing oxidative modification of cellular macromolecules, inhibiting protein function, and promoting cell death). The reactive sites present on MNPs surface make them capture electron easily, which can lead to the formation of the superoxide radical. And the superoxide radical can then generate additional ROS through dismutation or Fenton chemistry [54].

Although some studies have not found the direct correlation between toxicity and ROS production induced by MNPs [49], most studies concluded that nanoparticles can 
be toxic, not only affecting cells in a direct way, but also indirectly by the induction of excess ROS [54,55]. It is reported that toxicity of SPIONs with a mean size of $30 \mathrm{~nm}$ coated with Tween 80 surfactant in murine macrophage (J774) cells was found to be due to induction of oxidative stress and subsequent apoptosis. The intracellular ROS generation evaluated by a $\mathrm{H}_{2}$ DCFDDA assay showed both concentration- and time-dependent, and the enhanced ROS production led to cell injury and death [55].

Changes in protein/gene expression can reveal toxicity-dependent modulation of protein/gene expression and help to elucidate possible mechanisms of toxicity. In the study of cytotoxicity evaluation of various MNPs on the employed three human cell lines, it was revealed by the specific gene alteration and hierarchical clustering that SPIONs-COOH altered genes associated with cell proliferative responses due to their ROS properties [52]. The global gene expression of mouse macrophage RAW264.7 cells treated with MNPs coated with DMSA synthesized by our group demonstrated that this nanoparticle can strongly activate inflammatory and immune responses and can inhibit the biosynthesis and metabolism of RAW264.7 cells at a dose of $100 \mu \mathrm{g} / \mathrm{mL}$ [56]. The result is quite in line with the findings that phagocytic cells will release inflammatory factors when they uptake foreign materials. The cellular inflammation can also be assessed through qualitative observation of inflammatory protein expression by immunofluorescence or quantification of relevant protein signaling molecules (cytokines) at the protein level via enzyme-linked imunosorbent assay (ELLSA) or Western blotting. In the study of fluorescent-magnetic hybrid nanoparticles (ironplatinum embedded), a particle-dose-dependent increase of cytokine tumor necrosis factor alpha (TNF $\alpha$ ) was found in both cell types of the defense system but not in epithelial cells [8]. Other signalings were also studied. For one, high intracellular iron oxide nanoparticle concentrations can affect the actin cytoskeleton and the formation and maturation of focal adhesion complex (FAC), and can affect focal adhesion kinase (FAK) expression levels. The lower amount of FAK and the reduced stimuli through FACs can further affect the actin cytoskeleton architecture and reduce cell proliferation, and could have detrimental effects on cell migration and differentiation through FAK-mediated signaling pathway [45].

\section{Summary}

The in vitro biological effects of MNPs, especially cell responses to MNPs, as well as the interactions between nanoparticles with proteins from biological fluids, are highlighted in this article. Some effects have been concluded. However, because of the great variety in types of MNPs, cells and incubation protocols, it is impossible to make any conclusions regarding the safety of MNPs for biomedical im- plication. At nontoxic concentrations of MNPs, several of them might not be well suited for their original purpose, so it is important for the cytotoxicity assessments to evaluate MNPs efficiency, as well as the deep understanding of the toxicity pathways of various MNPs. Thus a great amount of reliable researches remain to be conducted for the safe and efficient use of MNPs in biomedical implications.

This work was supported by the National Basic Research Program of China (2011CB933503), the International Science and Technology Cooperation Program of China (2008DFA51180 and 2009DFA31990), and the Basic Research Program of Jiangsu Province (BK2009013 and BK2011036).

1 Pouponneau P, Leroux J C, Soulez G, et al. Co-encapsulation of magnetic nanoparticles and doxorubicin into biodegradable microcarriers for deep tissue targeting by Vascular Mri Navigation. Biomaterials, 2011, 32: 3481-3486

2 Lee J S, Rodriguez-Luccioni H L, Mendez J, et al. Hyperthermia induced by magnetic nanoparticles improves the effectiveness of the anticancer drug cis-diamminedichloroplatinum. J Nanosci Nanotechnol, 2011, 11: 4153-4157

3 Wilhelm C, Gazeau F. Universal cell labelling with anionic magnetic nanoparticles. Biomaterials, 2008, 29: 3161-3174

4 Subramanian N, Abimanyu S, Vinoth J, et al. Biodegradable chitosan magnetic nanoparticle carriers for sub-cellular targeting delivery of artesunate for efficient treatment of breast cancer. In: Hafeli U S W Z M, ed. 8th International Conference on the Scientific and Clinical Applications of Magnetic Carriers, 2010. 416-424

5 Roca A G, Costo R, Rebolledo A F, et al. Progress in the preparation of magnetic nanoparticles for applications in biomedicine. J Phys D-Appl Phys, 2009, 42: 224002

6 Berry C C. Progress in functionalization of magnetic nanoparticles for applications in biomedicine. J Phys D-Appl Phys, 2009, 42: 224003

7 Chen Z P, Zhang Y, Zhang S, et al. Preparation and characterization of water-soluble monodisperse magnetic iron oxide nanoparticles via surface double-exchange with Dmsa. Colloids and Surfaces. Physicochem Eng Aspects, 2008, 316: 210-216

8 Lehmann A D, Parak W J, Zhang F, et al. Fluorescent-magnetic hybrid nanoparticles induce a dose-dependent increase in proinflammatory response in lung cells in vitro correlated with intracellular localization. Small, 2010, 6: 753-762

9 Lewin M, Carlesso N, Tung $\mathrm{C} \mathrm{H}$, et al. Tat peptide-derivatized magnetic nanoparticles allow in vivo tracking and recovery of progenitor cells. Nat Biotechnol, 2000, 18: 410-414

10 Pirko I, Johnson A, Ciric B, et al. In vivo magnetic resonance imaging of immune cells in the central nervous system with superparamagnetic antibodies. FASEB J, 2003, 17: 179

11 Yang F, Li Y, Chen Z, et al. Superparamagnetic iron oxide nanoparticle-embedded encapsulated microbubbles as dual contrast agents of magnetic resonance and ultrasound imaging. Biomaterials, 2009, 30: 3882-3890

12 Kang J S, Yum Y N, Park S N. Cytotoxicity and DNA damage induced by magnetic nanoparticle silica in L5178y cell. Biomolecul Therapeutics, 2011, 19: 261-266

13 Lu Q H, Yao K L, Xi D, et al. Synthesis and characterization of composite nanoparticles comprised of gold shell and magnetic core/cores. J Magnet Magnet Mat, 2006, 301: 44-49

14 Walczyk D, Bombelli F B, Monopoli M P, et al. What the cell "sees" in bionanoscience. J Am Chem Soc, 2010, 132: 5761-5768

15 Verma A, Stellacci F. Effect of surface properties on nanoparticle - cell interactions. Small, 2010, 6: 12-21

16 Tenzer S, Docter D, Rosfa S, et al. Nanoparticle size is a critical physicochemical determinant of the human blood plasma corona: A comprehensive quantitative proteomic analysis. ACS Nano, 2011, 5: 7155-7167 
17 Lundqvist M, Stigler J, Cedervall T, et al. The evolution of the protein corona around nanoparticles: A test study. ACS Nano, 2011, 5: 7503-7509

18 Prapainop K, Wentworth P. A shotgun proteomic study of the protein corona associated with cholesterol and atheronal-B surface-modified quantum dots. Eur J Pharm Biopharm, 2011, 77: 353-359

19 Sund J, Alenius H, Vippola M, et al. Proteomic characterization of engineered nanomaterial-protein interactions in relation to surface reactivity. ACS Nano, 2011, 5: 4300-4309

20 Casals E, Pfaller T, Duschl A, et al. Time evolution of the nanoparticle protein corona. ACS Nano, 2010, 4: 3623-3632

21 Mahmoudi M, Serpooshan V. Large protein absorptions from small changes on the surface of nanoparticles. J Phys Chem C, 2011, 115: 18275-18283

22 Capriotti A L, Caracciolo G, Cavaliere C, et al. Shotgun proteomic analytical approach for studying proteins adsorbed onto liposome surface. Anal Bioanal Chem, 2011, 401: 1195-1202

23 Lundqvist M, Stigler J, Elia G, et al. Nanoparticle size and surface properties determine the protein corona with possible implications for biological impacts. Proc Natl Acad Sci USA, 2008, 105: 1426514270

24 Gessner A, Lieske A, Paulke B R, et al. Influence of surface charge density on protein adsorption on polymeric nanoparticles: Analysis by two-dimensional electrophoresis. Eur J Pharm Biopharm, 2002, 54: $165-170$

25 Chen $\mathrm{Z}, \mathrm{Xu} \mathrm{R}$, Zhang $\mathrm{Y}$, et al. Effects of proteins from culture medium on surface property of silanes-functionalized magnetic nanoparticles. Nanoscale Res Lett, 2008, 4: 204-209

26 Cedervall $\mathrm{T}$, Lynch I, Lindman S, et al. Understanding the nanoparticle-protein corona using methods to quantify exchange rates and affinities of proteins for nanoparticles. Proc Natl Acad Sci USA, 2007, 104: 2050-2055

27 Jones C F, Grainger D W. In vitro assessments of nanomaterial toxicity. Adv Drug Deli Rev, 2009, 61: 438-456

28 Huang J, Bu L, Xie J, et al. Effects of nanoparticle size on cellular uptake and liver Mri with polyvinylpyrrolidone-coated iron oxide nanoparticles. ACS Nano, 2010, 4: 7151-7160

29 Ge Y, Zhang Y, Xia J, et al. Effect of surface charge and agglomerate degree of magnetic iron oxide nanoparticles on $\mathrm{Kb}$ cellular uptake in vitro. Colloi Surfaces B-Biointerfaces, 2009, 73: 294-301

30 Ge Y, Zhang Y, He S, et al. Fluorescence modified chitosan-coated magnetic nanoparticles for high-efficient cellular imaging. Nanoscale Res Lett, 2009, 4: 287-295

31 Shi X, Thomas T P, Myc L A, et al. Synthesis, characterization, and intracellular uptake of carboxyl-terminated poly(amidoamine) dendrimer-stabilized iron oxide nanoparticles. Phys Chem Chem Phys, 2007, 9: 5712-5720

32 Zhang S, Chen X, Gu C, et al. The effect of iron oxide magnetic nanoparticles on smooth muscle cells. Nanoscale Res Lett, 2009, 4: 70-77

33 Liu $\mathrm{D}, \mathrm{Wu} \mathrm{W}$, Ling $\mathrm{J}$, et al. Effective pegylation of iron oxide nanoparticles for high performance in vivo cancer imaging. Adv Funct Mater, 2011, 21: 1498-1504

34 Josephson L, Tung C H, Moore A, et al. High-efficiency intracellular magnetic labeling with novel superparamagnetic-Tat peptide conjugates. Bioconjug Chem, 1999, 10: 186-191

35 Zhao M, Kircher M F, Josephson L, et al. Differential conjugation of Tat peptide to superparamagnetic nanoparticles and its effect on cellular uptake. Bioconjug Chem, 2002, 13: 840-844

36 Dodd C H, Hsu H C, Chu W J, et al. Normal T-cell response and in vivo magnetic resonance imaging of $\mathrm{T}$ cells loaded with HIV transactivator-peptide-derived superparamagnetic nanoparticles. J
Immunol Methods, 2001, 256: 89-105

37 Saha K, Bajaj A, Duncan B, et al. Beauty is skin deep: A surface monolayer perspective on nanoparticle interactions with cells and biomacromolecules. Small, 2011, 7: 1903-1918

38 Thomas M, Klibanov A M. Enhancing polyethylenimine's delivery of plasmid DNA into mammalian cells. Proc Nat Acad Sci USA, 2002, 99: 14640-14645

39 Rosi N L, Giljohann D A, Thaxton C S, et al. Oligonucleotidemodified gold nanoparticles for intracellular gene regulation. Science, 2006, 312: 1027-1030

40 Lin X, Li Y, Gu N. Nanoparticl's size effect on its translocation across a lipid bilayer: A molecular dynamics simulation. J Compu Theoret Nanosci, 2010, 7: 269-276

41 Li Y, Chen X, Gu N. Computational investigation of interaction between nanoparticles and membranes: Hydrophobic/hydrophilic effect. J Phys Chem B, 2008, 112: 16647-16653

42 Li Y, Gu N. Thermodynamics of charged nanoparticle adsorption on charge-neutral membranes: A simulation study. J Phys Chem B, 2010, 114: 2749-2754

43 Ding J, Tao K, Li J, et al. Cell-specific cytotoxicity of dextranstabilized magnetite nanoparticles. Colloi Surfaces B-Biointerfaces, 2010, 79: 184-190

44 Soenen S J H, Himmelreich U, Nuytten N, et al. Cytotoxic effects of iron oxide nanoparticles and implications for safety in cell labelling. Biomaterials, 2011, 32: 195-205

45 Soenen S J H, Nuytten N, De Meyer S F, et al. High intracellular iron oxide nanoparticle concentrations affect cellular cytoskeleton and focal adhesion kinase-mediated signaling. Small, 2010, 6: 832842

46 Villanueva A, Canete M, Roca A G, et al. The influence of surface functionalization on the enhanced internalization of magnetic nanoparticles in cancer cells. Nanotechnology, 2009, 20:

47 Xia J G, Zhang S, Zhang Y, et al. Maghemite nanoparticles and their protamine derivatives: Cellular internalization and effects on cellcycle progress. J Nanosci Nanotechnol, 2009, 9: 1025-1028

48 Ankamwar B, Lai T C, Huang $\mathrm{J} \mathrm{H}$, et al. Biocompatibility of $\mathrm{Fe}_{3} \mathrm{O}_{4}$ nanoparticles evaluated by in vitro cytotoxicity assays using normal, glia and breast cancer cells. Nanotechnology, 2010, 21: 075102

49 Diaz B, Sanchez-Espinel C, Arruebo M, et al. Assessing methods for blood cell cytotoxic responses to inorganic nanoparticles and nanoparticle aggregates. Small, 2008, 4: 2025-2034

50 Ito $\mathrm{A}$, Hibino $\mathrm{E}$, Honda $\mathrm{H}$, et al. A new methodology of mesenchymal stem cell expansion using magnetic nanoparticles. Biochem Eng J, 2004, 20: 119-125

51 Cotica L F, Freitas V F, Dias G S, et al. Simple and facile approach to synthesize magnetite nanoparticles and assessment of their effects on blood cells. J Magnet Magnet Mater, 2012, 324: 559-563

52 Mahmoudi M, Laurent S, Shokrgozar M A, et al. Toxicity evaluations of superparamagnetic iron oxide nanoparticles: Cell "vision" versus physicochemical properties of nanoparticles. ACS Nano, 2011, 5: 7263-7276

53 Liu Y, Wang J. Comparative and quantitative investigation of cell labeling of a 12-Nm Dmsa-coated $\mathrm{Fe}_{3} \mathrm{O}_{4}$ magnetic nanoparticle with multiple mammalian cell lines. J Mater Res, 2011, 26: 822-831

54 Nel A, Xia T, Madler L, et al. Toxic potential of materials at the nanolevel. Science, 2006, 311: 622-627

55 Naqvi S, Samim M, Abdin M Z, et al. Concentration-dependent toxicity of iron oxide nanoparticles mediated by increased oxidative stress. Inter J Nanomed, 2010, 5: 983-989

56 Liu Y, Chen Z, Gu N, et al. Effects of Dmsa-coated $\mathrm{Fe}(3) \mathrm{O}(4)$ magnetic nanoparticles on global gene expression of mouse macrophage Raw264.7 cells. Toxicol Lett, 2011, 205: 130-139

Open Access This article is distributed under the terms of the Creative Commons Attribution License which permits any use, distribution, and reproduction in any medium, provided the original author(s) and source are credited. 